\title{
Capacidad Vesical como Pronóstico de Trasplante Renal
}

\section{Bladder Capacity as a Renal Transplant Prognosis}

\author{
William Ricardo Fajardo Cediel ${ }^{1}$ Karen Viviana Flórez Barbosa ${ }^{1}$ Jenny Catherin García Cuevas ${ }^{2}$ \\ María Paula Rodríguez ${ }^{2}$ Mónica María Zúñiga Castrillón ${ }^{3}$ Carolina Andrea Duarte Gonzalez ${ }^{4}$
}

\footnotetext{
${ }^{1}$ Grupo Trasplante Fundación Clínica Shaio, Bogotá D.C., Colombia

2 Universidad de La Sabana, Chía Cundinamarca, Colombia

3 Medicina Crítica y Cuidado Intensivo, Universidad de La Sabana, Chía Cundinamarca, Colombia

4 Pontificia Universidad Javeriana, Bogotá D.C., Colombia
}

Address for correspondence Grupo Trasplante, Fundación Clínica Shaio Diagonal 115 A No. 70 C-30 Bogotá D.C., Colombia (e-mail: transplante@shaio.org).

Urol Colomb 2020;29:32-38.

\section{Resumen}

Palabras clave

- capacidad vesical

- trasplante renal

- terapia de reemplazo renal

- complicaciones urológicas
Introducción La capacidad vesical pre trasplante renal, ha demostrado tener un impacto significativo en el pronóstico, la calidad de vida y complicaciones urológicas en los pacientes.

Objetivo Estimar la correlación de Pearson que existe entre la capacidad vesical pretrasplante (CVPRE) comparado con el tiempo de duración de Terapia de reemplazo renal (TRR); como factor pronóstico.

Métodos Se realizó una base de datos de pacientes con Trasplante Renal realizados por un Grupo Trasplante, de una sola institución, en los últimos 7 años, desde el 2010 hasta el año 2018. Se tomaron datos del estudio pre trasplante por parte del servicio de Urología y controles mensuales pos trasplante, hasta la fecha. Se tomaron en cuenta datos como: Tiempo de diagnóstico de ERC estadio final, tiempo y tipo de terapia de reemplazo renal, capacidad vesical y función renal pre-trasplante versus función renal y capacidad vesical pos-trasplante y complicaciones urológicas pos-trasplante. Con esos datos, se realizó el cruce de variables, utilizando el Coeficiente de correlación de Pearson, expresando resultados con valores entre 1, para mostrar correlación y significancia estadística en la práctica clínica.

Resultados Entre los años 2010 hasta mayo de 2018, se llevaron a cabo 203 Trasplantes renales, se tabularon 114 pacientes cuyos datos se encontraban completos. La Correlación Pearson fue de: $-0,3$ entre tiempo de TRR y CVPRE; $-0,14$ entre CVPRE y creatinina pos TR; 0,09 entre CVPRE y RVU; de 0,14 entre CVPRE e IVU; esas 2 últimas, complicaciones urológicas pos-trasplante renal.

Conclusiones Existe correlación inversa entre el tiempo de TRR y Capacidad vesical pretrasplante, lo cual influye en el pronóstico del paciente trasplantado renal, en términos de función renal pos-trasplante y complicaciones urológicas dadas por RVU e IVU.

\section{Introducción}

El trasplante renal constituye la mejor terapia disponible para los pacientes con enfermedad renal crónica en estadio

received

November 29, 2018

accepted

July 19, 2019
DOI https://doi.org/

$10.1055 / \mathrm{s}-0039-1696698$.

ISSN 0120-789X.

e ISSN 2027-0119. terminal, sin embargo, las complicaciones urológicas posteriores a su realización son frecuentes y se relacionan con importante morbilidad y mortalidad. ${ }^{1}$ Entre las

Copyright $\odot$ 2020, Sociedad Colombiana License terms de Urología. Publicado por Thieme Revinter Publicações Ltda., Rio de Janeiro, Brazil. Todos los derechos reservados. 
Abstract

\section{Keywords}

- bladder capacity

- kidney transplant

- renal replacement therapy

- urological complications
Introduction The bladder capacity has demonstrated to have an important impact in prognosis, quality of life and urological complications in patients.

Objective Estimate the Pearson correlation that exists between pre-transplant bladder capacity compared to the duration of renal replacement therapy (RRT); as a prognosis factor.

Methods A database of Kidney Transplantation patients performed by a Transplant Group, from a single institution, during the last 7 years, from 2010 to 2018, was made. Data from the pretransplant study carried out by the Urology Service and from the posttransplant monthly controls, to date were also analyzed. Data such as: Time of CKD final stage diagnosis, time and type of renal replacement therapy, bladder capacity and pretransplant renal function versus post-transplant renal function and bladder capacity and post-transplant urological complications, were also taken into consideration. With these data, a variable crossing was carried out, using the Pearson Correlation Coefficient, expressing results with values ranging between -1 and 1 , to show correlation and statistical significance in clinical practice.

Results 203 kidney transplants carried out between years 2010 to May 2018; of these 114 patients, whose data were complete, were tabulated. The Pearson Correlation was: -0.3 between RRT and PTBC time; -0.14 between PTBC and creatinine after TR; 0.09 between PTBC and RVU; of 0.14 between PTBC and IVU; the last 2 were urological complications after kidney transplant.

Conclusions There is an inverse correlation between RRT Time and bladder capacity before transplantation, which influences the prognosis of the renal transplant patient, in terms of post-transplant renal function and urological complications due to VUR and UTI. complicaciones urológicas más frecuentes se encuentran; estenosis ureteral, reflujo vesicoureteral (RVU), fugas urinarias e infecciones de vías urinarias (IVU). ${ }^{1}$

La mayoría de los pacientes que son llevados a trasplante renal, han permanecido en terapia de reemplazo renal durante largos periodos de tiempo lo que lleva a una atrofia vesical y perdida de la distensión fisiológica normal severa secundaria a oligoanuria, generando finalmente a una disminución de la compliance y capacidad vescial. ${ }^{2,3}$

A pesar de que se restablezca el gasto urinario normal posterior a la realización del trasplante renal, ya se han generado cambios anatómicos y funcionales del tracto urinario inferior que favorecen con facilidad la aparición de polaquiuria, urgencia, incontinencia de urgencia, reflujo vesicoureteral e infección de vías urinarias que disminuye de forma importante la sobrevida de los pacientes trasplantados, y que también influye en la sobrevida del injerto. ${ }^{1}$

Actualmente, se encuentran estudios que relacionan el tiempo de diálisis con la aparición de complicaciones posterior a la realización del trasplante renal, sin embargo, no se encuentran estudios en los que se relacione la capacidad vesical con la aparición de esas complicaciones, por lo que se llevó a cabo un estudio retrospectivo con el fin de Estimar la correlación de Pearson que existe entre la capacidad vesical pretrasplante (CVPRE) comparado con el tiempo de duración de Terapia de reemplazo renal (TRR); como factor pronóstico Se obtuvo una muestra de 114 pacientes trasplantados en una institución de IV nivel desde Enero de 2010 hasta Mayo de 2018.
El objetivo del presente estudio fue estimar la correlación de Pearson que existe entre la capacidad vesical pretrasplante (CVPRE) comparado con el tiempo de duración de Terapia de reemplazo renal (TRR); como factor pronóstico.

\section{Métodos}

Se revisó la historia clínica de todos los pacientes llevados a trasplante renal entre enero de 2010 y mayo de 2018 en una institución de cuarto nivel en Bogotá, encontrando un total de 203 pacientes trasplantados, donde únicamente se incluyeron 114 dentro del estudio ya que los 89 restantes no contaban con toda la información requerida. Las características de la población estudiada se encuentran en la - Tabla 1, con las variables utilizadas en la recolección de datos como sexo, edad, tiempo de TRR menor o igual a 5 años vs mayor a 5 años, punto de corte utilizado como facilidad en el momento de la clasificación de la información, capacidad vesical pretrasplante, función renal, y si el paciente se encontraba en anuria o no previo al trasplante renal. En ese último aspecto resaltamos que, en las historias clínicas, no se registró el tiempo de anuria de esos pacientes, por lo cual el tiempo no se incluye dentro del estudio. El método utilizado para la medición de la capacidad vesical pre-trasplante en los pacientes del estudio, fue la cistoscopia pre-trasplante, razón por la cual, es relevante el registro de ese dato.

Adicionalmente, se analizó la relación entre la capacidad vesical pre-trasplante con la aparición de complicaciones 
Tabla 1 Características de la muestra y variables utilizadas

\begin{tabular}{|c|c|c|}
\hline Variable & Característica & Valor \\
\hline \multirow[t]{2}{*}{ Edad Promedio } & Hombre & 49 (años) \\
\hline & Mujer & 43 (años) \\
\hline \multirow[t]{2}{*}{ Sexo } & Femenino & 35 (Pacientes) \\
\hline & Masculino & 79 (Pacientes) \\
\hline \multirow[t]{2}{*}{ Tiempo de Diálisis } & $\leq 5$ años & 83 (Pacientes) \\
\hline & $>5$ años & 31 (Pacientes) \\
\hline \multirow[t]{2}{*}{ Anuria } & Sí & 46 (Pacientes) \\
\hline & No & 68 (Pacientes) \\
\hline \multirow[t]{2}{*}{ Diabéticos } & Sí & 10 (Pacientes) \\
\hline & No & 104 (Pacientes) \\
\hline \multirow{2}{*}{$\begin{array}{l}\text { Cistoscopia } \\
\text { Pre-trasplante }\end{array}$} & Sí & 14 (Pacientes) \\
\hline & No & 100 (Pacientes) \\
\hline \multirow{3}{*}{$\begin{array}{l}\text { Capacidad Vesical } \\
\text { Pre-trasplante }\end{array}$} & Máximo & $500(\mathrm{~mL})$ \\
\hline & Mínimo & $50(\mathrm{~mL})$ \\
\hline & Promedio & $350,6(\mathrm{~mL})$ \\
\hline \multirow{2}{*}{$\begin{array}{l}\text { Hidrodistensión o } \\
\text { antimuscarínicos }\end{array}$} & Sí & 8 (Pacientes) \\
\hline & No & 106 (Pacientes) \\
\hline \multirow[t]{2}{*}{ RVU Pre-trasplante } & Sí & 7 (Pacientes) \\
\hline & No & 107 (Pacientes) \\
\hline \multirow[t]{2}{*}{ Donante } & Cadavérico & 98(Pacientes) \\
\hline & Vivo & 16 (Pacientes) \\
\hline Creatinina al 1 Mes & Promedio & $1,4(\mathrm{mg} / \mathrm{dL})$ \\
\hline \multirow{3}{*}{$\begin{array}{l}\text { Creatinina al Año } \\
\text { Pos-trasplante }\end{array}$} & Máximo & $10,9(\mathrm{mg} / \mathrm{dL})$ \\
\hline & Mínimo & $0,7(\mathrm{mg} / \mathrm{dL})$ \\
\hline & Promedio & $1,58(\mathrm{mg} / \mathrm{dL})$ \\
\hline \multirow{2}{*}{$\begin{array}{l}\text { Cistoscopia Pos- } \\
\text { trasplante }\end{array}$} & Sí & 4 (Pacientes) \\
\hline & No & 110(Pacientes) \\
\hline \multirow{3}{*}{$\begin{array}{l}\text { Capacidad Vesical } \\
\text { Pos-trasplante }\end{array}$} & Máximo & $500(\mathrm{~mL})$ \\
\hline & Mínimo & $100(\mathrm{~mL})$ \\
\hline & Promedio & $415,2(\mathrm{~mL})$ \\
\hline \multirow{2}{*}{$\begin{array}{l}\text { Síntomas Urinarios } \\
\text { Irritativos Pos- } \\
\text { trasplantes: (Disuria, } \\
\text { tenesmo vesical y/o } \\
\text { polaquiuria) }\end{array}$} & Sí & 20 (Pacientes) \\
\hline & No & 94(Pacientes) \\
\hline \multirow{2}{*}{$\begin{array}{l}\text { RVU } \\
\text { pos-trasplante }\end{array}$} & Sí & 4 (Pacientes) \\
\hline & No & 110 (Pacientes) \\
\hline \multirow[t]{2}{*}{ Infecciones } & Sí & 4 (Pacientes) \\
\hline & No & 110 (Pacientes) \\
\hline \multirow{2}{*}{$\begin{array}{l}\text { Fistulas } \\
\text { Pos-trasplante }\end{array}$} & Sí & 0 (Pacientes) \\
\hline & No & 114 (Pacientes) \\
\hline
\end{tabular}

urológicas hasta la fecha de revisión de las historias clínicas, y con la función renal posterior a un año de la realización del trasplante. Adicionalmente se evaluó la relación entre el tiempo de terapia de reemplazo renal con la capacidad vesical previa al trasplante.
Previo a la realización del trasplante renal, todos los pacientes fueron sometidos a cistografía como método de medición de la capacidad vesical. La función renal previa y posterior al trasplante renal fue tomada a partir de la creatinina sérica.

Para ese estudio, se tienen en cuenta las complicaciones urológicas más frecuentes presentadas en los pacientes llevados a Trasplante Renal. Tales complicaciones fueron: infección de vías urinarias, aparición de reflujo vesicoureteral y fistulas.

Se realizaron los 4 cruces de variables con la Correlación de Pearson, utilizando para eso el programa Excel y se graficaron en gráficas de dispersión, las tendencias de los resultados. Los cruces de variables fueron: Tiempo de Terapia de Reemplazo Renal y Capacidad vesical Pretrasplante (- Figura 1), Capacidad vesical Pre-trasplante y Valor de Creatinina al año (-Figura 2), Capacidad vesical Pre-trasplante y Reflujo Vesicoureteral (-Figura 3) y finalmente, capacidad vesical pre-trasplante e Infección de vías Urinarias (-Figura 4).

\section{Discusión}

Al momento de revisar la literatura, con respecto al pronóstico de trasplante renal, basados en la capacidad vesical pre trasplante (CVPRE), no se encontraron estudios clínicos con respecto a esta relación estadística. A continuación mencionamos estudios que pueden inferir en esta relación en los pacientes llevados a Trasplante renal. Posteriormente hacemos una correlación con los hallazgos encontrados en el nuestro grupo trasplante estudiado.

En Japón, realizaron un estudio clínico con una muestra de 408 pacientes, evidenciando que la reducción en la capacidad vesical debido a diálisis y anuria, está en correlación directa con la duración de la diálisis. ${ }^{4}$ La atróa vesical en los pacientes con trasplante renal después de la diálisis a largo plazo, está asociada con un mayor riesgo de

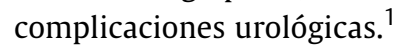

Otro Estudio de 622 pacientes, con diagnóstico de Enfermedad Renal Crónica, estadio final que se encontraban en diálisis por periodos de tiempo largos, van a tener mayor probabilidad de presentar capacidad vesical reducida y reflujo vesicoureteral. Sin embargo, la capacidad vesical en sí misma, no está relacionada con el desarrollo de disfunción del tracto urinario inferior. ${ }^{5}$ La capacidad vesical reducida y la compliance en los pacientes con ERC, puede afectar el almacenamiento y la función miccional posterior al trasplante renal. ${ }^{2}$ Se han comparando esas variables de Tiempo de TRR y capacidad vesical con muestra en 101 pacientes, evidenció que a pesar de que la capacidad vesical disminuyó debido a la diálisis de periodos largos, sobrepasó $150 \mathrm{~mL}$ al 1 año pos trasplante renal. Una vejiga pequeña puede ser utilizada en trasplante renal, pero puede aumentar el riesgo de reflujo vesicoureteral. ${ }^{6}$

La realización de cistometría preoperatoria y postoperatoria para la valoración de la función vesical, ${ }^{7}$ evidencia una mejoría significativa en sus parámetros y en el estudio de presión de flujo, observados en los pacientes con enfermedad renal crónica en estadio final, sin patología 


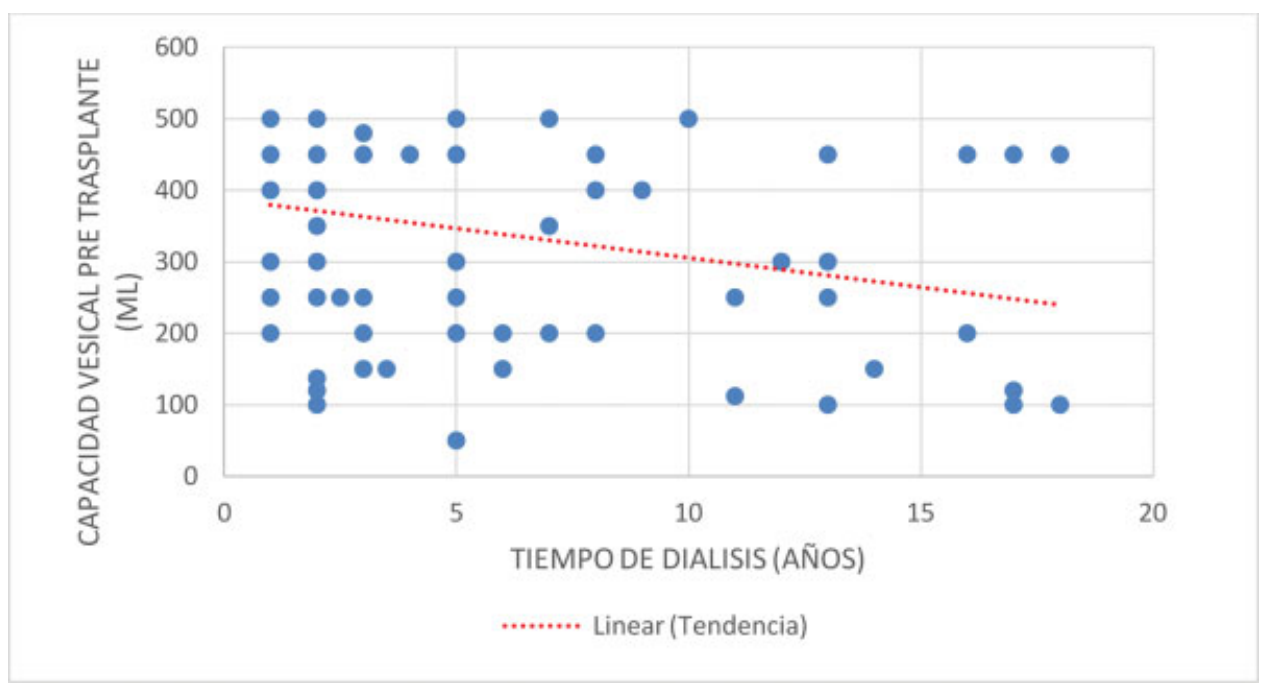

Fig. 1. Tiempo de Terapia de Reemplazo Renal y Capacidad vesical Pre-trasplante.

urológica 6 meses posteriores al trasplante renal. Eso estuvo asociado con la recuperación de la tasa de filtración glomerular y el gasto urinario, dados por el injerto renal. ${ }^{8}$ Finalmente, un estudio publicado en el 2016, con muestra de 61 pacientes sin patología neurogénica, que fueron llevados a video cistometría con agua pre y 1 año post trasplante, fueron estudiados, arrojando como resultado la duración de la diálisis pretrasplante. Ello se correlacionó con la capacidad de la vejiga pre-trasplante y compliance $(\mathrm{R} 2=0,421, \quad p<0,001 \quad \mathrm{y}$ $\mathrm{R} 2=0,418, p<0,001$, respectivamente); mostrando que la capacidad vesical disminuye logarítmicamente pretrasplante, de acuerdo con la duración de la diálisis. ${ }^{3}$

Con respecto a la función vesical, un estudio de 92 pacientes con un promedio de tiempo de TRR de 60,2 meses, la mayoría presentó disfunción vesical debido a: baja capacidad, baja compliance e hiperactividad del detrusor. ${ }^{9}$ Adicionalmente, la presencia de sintomatología del tracto urinario inferior pos-trasplante, se asocia con la capacidad vesical reducida, evidenciado en estudio de 52 pacientes llevados a trasplante en el 2008. ${ }^{10}$

En la revisión de la literatura, se encontraron artículos con respecto a la necesidad del uso de medidas terapéuticas para el aumento dela capacidad vesical pre-trasplante y los resultados de los mismos. ${ }^{11}$ En el 2008, se estudiaron 345 pacientes retrospectivamente, con ERC, llevados a trasplante renal entre el 2002 y 2006, seleccionándolos en Grupo A con capacidad vesical menor de $100 \mathrm{~mL}$ y Grupo B con capacidad vesical mayor de $100 \mathrm{~mL}$. Como resultado, no hubo diferencia en la frecuencia de complicaciones quirúrgicas y de infecciones del tracto urinario, ni en el número de hospitalizaciones entre los 2 grupos, así mismo, no hubo diferencia estadística entre los grupos con respecto a los

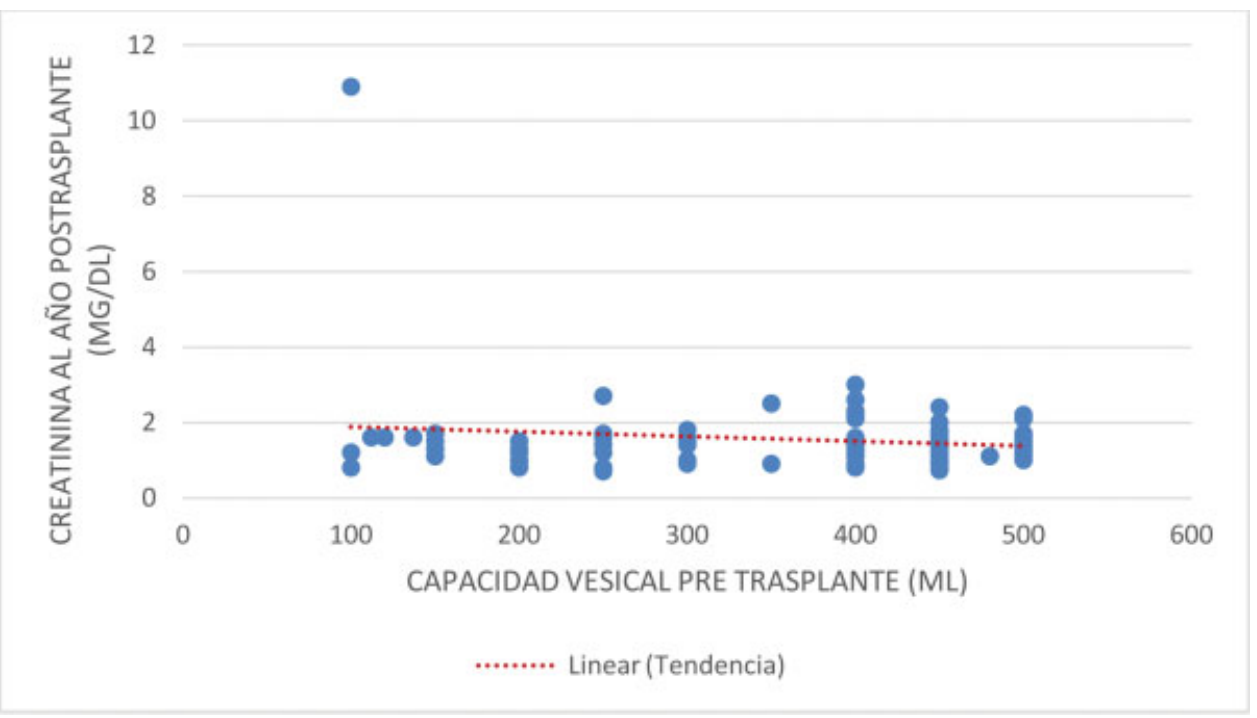

Fig. 2. Capacidad vesical Pre-trasplante y Valor de Creatinina al año. 


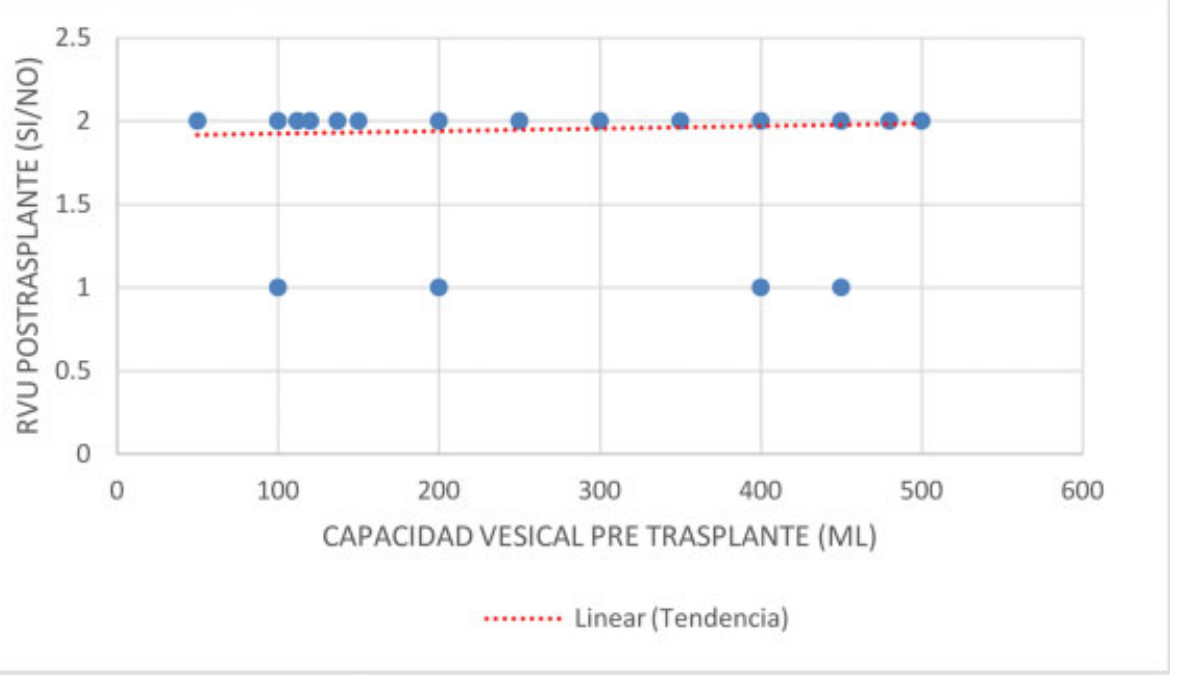

Fig. 3. Capacidad vesical Pre-trasplante y Reflujo Vesicoureteral.

niveles de creatinina sérica y la sobrevida del injerto a los 5 años posteriores al trasplante (100\% vs $92,4 \%)^{12}$

Con respecto al aumento vesical por medio de cistoplastia pre o pos trasplante, durante un periodo de seguimiento de 20 años, se obtuvo una muestra de 1406 pacientes en Irán, con un brazo en Grupo A: a los cuales se les realizo enteroplastia y al Grupo B: se les realizó ureterocistoplastia concluyendo que ambos procedimientos son igualmente recomendados antes del trasplante renal para la reconstrucción del tracto urinario inferior. $^{13}$

El tema de hidrodistención vesical, tuvo lugar en el Journal de Escandinavia del 2016, se publicó articulo con 6 pacientes que presentaban capacidad vesical menor de $100 \mathrm{~mL}$, con una media pre hidrodistención de $40 \mathrm{~mL}$ y aumento a $200 \mathrm{~mL}$ dentro de las primeras 2 semanas posteriores a la terapia con BCG, concluyendo que la hidrositención vesical es un tratamiento efectivo en pacientes con disminución severa de la capacidad vesical. ${ }^{14}$ Así mismo, el manejo con antimuscarinicos (Solifenacina) para mejorar la disfunción del tracto urinario pos-trasplante renal, en un estudio de 33 pacientes donde un grupo de pacientes no fue tratado con antimuscarínicos y el grupo 2 sí; mostró en la cistometría, un aumento significativo en la capacidad cistometríca máxima en el grupo 2 que en el grupo $1(173,0+-60,7 \mathrm{~mL}$ en el grupo 1 , y $260,1+-51,0 \mathrm{~mL}$ en el grupo $2[p=0,005])$ posterior al trasplante renal. ${ }^{15}$

La importancia del estudio y seguimiento de la capacidad vesical en pacientes pediátricos llevados a trasplante renal, fue evaluada con 27 pacientes Children's Kidney Center Barnabas Health. Seis pacientes presentaban anuria desde el nacimiento. Los pacientes fueron levados a diálisis peritoneal después de 78 días y fueron llevados a trasplante renal a los 23 meses. Evidenciando que los pacientes con anuria desde el nacimiento tenían una tasa más elevada de infecciones del tracto urinario y

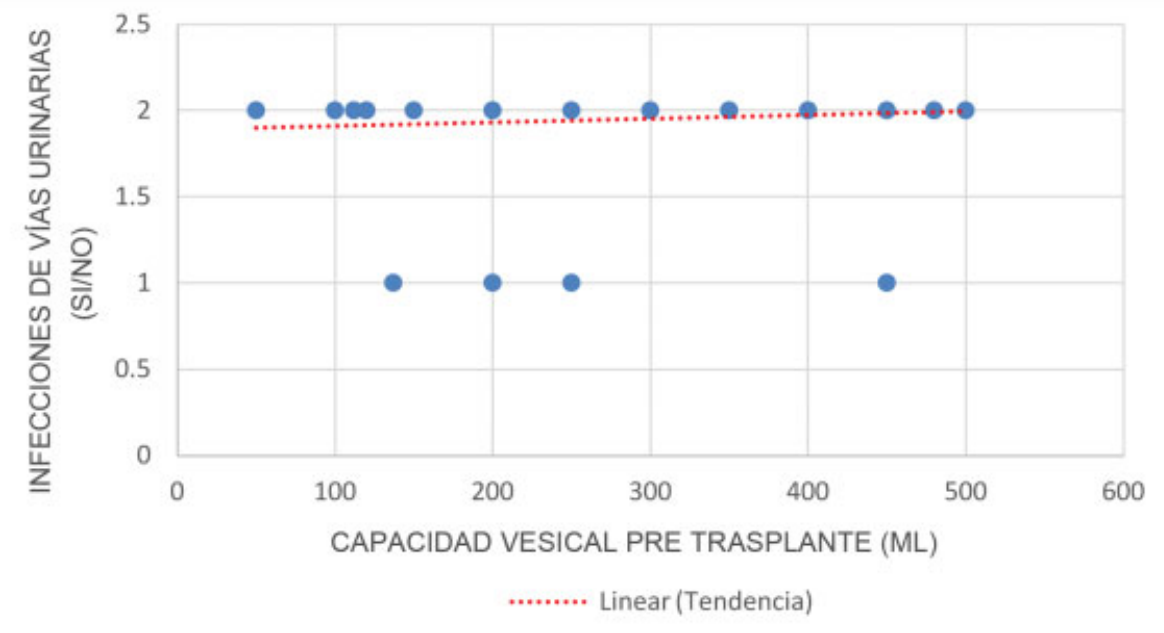

Fig. 4. Capacidad vesical pre-trasplante e Infección de vías Urinarias. 
de reflujo vesicoureteral que el grupo control (66\% vs 38\%). ${ }^{16}$ Adicionalmente, en un estudio retrospectivo desde el 2004 al 2008, 12 pacientes pediátricos con baja capacidad vesical y vejigas disfuncionales, presentan retos quirúrgicos únicos, ya que se concluye que es razonable realizar trasplante renal sin aumento vesical pre-trasplante, lo cual evita un procedimiento quirúrgico innecesario. ${ }^{17}$ En el 2010, 13 niños con vejiga valvular fueron llevados a trasplante renal $\mathrm{y}$ fueron considerados para aumento vesical. Todos presentaban oliguria al momento del trasplante; con seguimiento a los 12-3-4y 6 meses, los 5 niños que no fueron llevados a aumento vesical, mostraron función renal normal, con mejoría de la capacidad vesical y ausencia de hidronefrosis. Concluyendo que, en pacientes pediátricos, el trasplante renal puede ser realizado de manera segura sin requerir la necesidad de aumento vesical. ${ }^{18}$ Los niños con una reconstrucción vesical pueden ser llevados a trasplante renal con el mismo resultado de sobrevida del injerto y función del injerto, que aquellos con vejigas normales. ${ }^{19,20}$

Finalmente, se encontró un solo artículo en la búsqueda sobre el rol del Stent Ureteral en las ureteroneocistostomías en trasplantes renales, tema aislado al objetivo de este artículo, concluyendo la revisión de la literatura. ${ }^{21}$

Es claro entonces que el tiempo de duración de terapia de reemplazo renal está relacionado con la atrofia vesical, lo cual lleva por si mismo al desarrollo de diversas complicaciones mencionadas en los estudios anteriores. Esta información es muy similar a la encontrada en nuestro grupo de trasplantes.

Desde el 2010 hasta mayo de 2018, se llevaron a cabo 203 trasplantes renales, en una clínica de cuarto nivel en Bogotá, Colombia, se tabularon 114 pacientes cuyos datos se encontraban completos. La Correlación Pearson fue de: -0,3 entre tiempo de TRR y CVPRE; -0,14 entre CVPRE y creatinina pos TR; 0,09 entre CVPRE y RVU; de 0,14 entre CVPRE e IVU; esas 2 últimas fueron complicaciones urológicas pos-trasplante renal.

Eso indica, que las variables tiempo de TRR y CVPRE tienen una correlación inversa, indicando que a mayor tiempo de terapia de reemplazo renal, menor capacidad vesical pre trasplante se presenta, como lo La correlación de CVPRE y valor de creatinina pos trasplante renal tiene un valor de $R$ negativo, indicando la correlación inversa de esas dos variables; entre mayor capacidad vesical pre-trasplante, menor valor de creatinina pos-trasplante renal.

La presencia de complicaciones pos trasplante renal con respecto a la capacidad vesical pre-trasplante, da un valor positivo, teniendo en cuenta que las respuestas obtenidas era la presencia de no complicaciones, y que siendo positiva la respuesta, se asignó un valor de 1, y siendo negativa la respuesta valor de 2 . Teniendo en cuenta lo anterior, ese coeficiente es positivo indicando que a mayor capacidad vesical mayor respuesta negativa de los pacientes que presentaban complicaciones, es decir, que su respuesta fue que no las presentaron.

Como se mencionó anteriormente, no existen en la actualidad estudios que como este, que pretendan establecer la relación de la capacidad vesical pre trasplante con la aparición de complicaciones y mortalidad; sin embargo por los estudios previamente mencionados y los hallazgos de nuestro estudio es claro que la atrofia vesical se asocia con una disminución de la capacidad vesical, con aparición de varias complicaciones (en nuestro estudio dos en particular) lo cual incrementa la morbilidad en los pacientes llevados a Trasplante Renal.

Es importante tener en cuenta que en cada cruce de variables la muestra cambia debido a que no se encontraban datos de algunos pacientes, por lo tanto se eliminaron los resultados de cada paciente que no tuviera algún dato en cada par de variables a cruzar, se realizó de esa forma porque entre más datos en cada variable tenemos un estudio más consistente, por esa razón los resultados de cada cruce de variables no se pueden relacionar entre sí, debido a que el tamaño de la muestra en cada cruce es diferente.

\section{Conclusiones}

Existe correlación inversa entre el tiempo de TRR y Capacidad vesical pre-trasplante, lo cual influye en el pronóstico del paciente trasplantado renal, en términos de función renal pos-trasplante y complicaciones urológicas dadas por RVU e IVU.

A pesar de que se encuentra una relación importante entre la capacidad vesical pre trasplante y las complicaciones posterior a la realización del mismo, y por lo tanto pronóstico, es importante la realización de estudios posteriores, con mayor numero de pacientes con la finalidad de obtener un mayor número de datos que permitan establecer conclusiones que aporten de forma significativa en el pronostico de los paciente con trasplante renal.

Conflicto de Intereses

Los autores declaran no tener ningún conflicto de intereses.

\section{Referencias}

1 Hotta K, Miura M, Wada Y, et al. Atrophic bladder in long-term dialysis patients increases the risk for urological complications after kidney transplantation. Int J Urol 2017;24(04):314-319

2 Chen J-L, Lee M-C, Kuo H-C. Reduction of cystometric bladder capacity and bladder compliance with time in patients with end-stage renal disease. JFormos Med Assoc 2012;111(04): 209-213

3 Inoue T, Satoh S, Obara T, et al. Cystometric evaluation of recovery in hypocompliant defunctionalized bladder as a result of longterm dialysis after kidney transplantation. Int J Urol 2016;23(08): 694-700

4 Thuret R, Hurel S, Kleinclauss F, Timsit O. Lower urinary tract symptoms and pelvic floor dysfunction in renal transplant candidates and recipients. Prog Urol 2016;26(15):114-1121

5 Song M, Park J, Kim YH, et al. Bladder capacity in kidney transplant patients with end-stage renal disease. Int Urol Nephrol 2015;47 (01):101-106

6 Miyaoka R. Correlations between pretransplant dialysis duration, bladder capacity, and prevalence of vesicoureteral reflux to the graft. Int Braz J Urol 2011;37:551-552

7 Ciszek M. Urological evaluation prior to renal transplantation. Cent European J Urol 2015;68(03):383 
8 Silva DM, Prudente AC, Mazzali M, Borges CF, D’Ancona C. Bladder function evaluation before renal transplantation in nonurologic disease: is it necessary? Urology 2014;83(02): 406-410

9 Tsunoyama K, Ishida H, Omoto K, Shimizu T, Shirakawa H, Tanabe K. Bladder function of end-stage renal disease patients. Int J Urol 2010;17(09):791-795

10 Van der Weide MJ, Van Achterberg T, Smits JP, Heesakkers JP, Bemelmans BL, Hilbrands LB. Causes of frequency and nocturia after renal transplantation. BJU Int 2008;101(08):1029-1034

11 Blanco M, Medina J, Pamplona M, et al. Outcome of renal transplantation in adult patients with augmented bladders. Transplant Proc 2009;41(06):2382-2384

12 Chun JM, Jung GO, Park JB, et al. Renal transplantation in patients with a small bladder. Transplant Proc 2008;40(07): 2333-2335

13 Zafarghandi RM, Naghibi M, Nazemian F, Sharifipoor F, Taghavi R. 333 A Comparison Of Augmentation Enterocystoplastyand Ureterocystoplasty Before Kidney Transplantation With Control Group. Eur Urol Suppl 2010;9:130

14 Numakura K, Tsuchiya N, Tsuruta H, et al. Efficacy and safety of bladder hydrodistension for decreased bladder capacity induced by intravesical BCG therapy. Scand J Urol 2016;50 (06):429-432
15 Tsunoyama K, Ishida H, Shimizu T, Omoto K, Tanabe K. Improvement of urinary dysfunction after kidney transplantation by administration of the antimuscarinic agent-prospective randomized controlled study. Transplantation 2012;93(06):597-602

16 Filler G, Dave S. Is a reduction in cystometric bladder capacity in anuric infants post-renal transplant really no big deal? Pediatr Transplant 2016;20(08):1016-1017

17 Alexopoulos S, Lightner A, Concepcion W, Rose M, SalcedoConcepcion K, Salvatierra O. Pediatric kidney recipients with small capacity, defunctionalized urinary bladders receiving adult-sized kidney without prior bladder augmentation. Transplantation 2011;91(04):452-456

18 Capozza N, Torino G, Collura G, et al. Renal transplantation in patients with "valve bladder": is bladder augmentation necessary? Transplant Proc 2010;42(04):1069-1073

19 Pereira PL, Urrutia MJM, Lobato R, Jaureguizar E. Renal transplantation in augmented bladders. Curr Urol Rep 2014;15 (08):431

20 Herthelius M, Öborn $\mathrm{H}$. Bladder dysfunction in children and adolescents after renal transplantation. Pediatr Nephrol 2006; 21(05):725-728

21 Laftavi MR, Chaudhry Q, Kohli R, et al. The role of ureteral stents for all ureteroneocystostomies in kidney transplants. Int J Organ Transplant Med 2011;2(02):66-74 